\title{
Do Systemic Infections Contribute to the Pathogenesis of Dementia?
}

\author{
Keenan Sterling $^{1} \cdot$ Mengen Xing $^{2} \cdot$ Weihong Song ${ }^{2,3}$ (D)
}

Received: 27 August 2021 / Accepted: 20 October 2021/Published online: 1 December 2021

(C) Center for Excellence in Brain Science and Intelligence Technology, Chinese Academy of Sciences 2021

With over 50 million people currently living with dementia and numbers expected to double every twenty years, the world is currently facing a dementia epidemic. Nevertheless, a poor understanding of the etiology of dementia has resulted in the inability to develop any successful diseasemodifying treatment. Over the past year, several researchers have highlighted the role of systemic infections in increasing the risk of developing dementia. These findings are particularly troubling given the ongoing coronavirus 19 (COVID-19) pandemic, adding yet another cause for concern over the long-term impact of COVID-19 on the public's mental health.

In a recent article published in The Lancet Infection Diseases, Sipilä and colleagues investigated whether being hospitalized following a severe infection was linked with an increased risk of developing dementia [1]. Previous findings have suggested that people who experienced a severe infectious disease are more likely to be diagnosed with dementia in subsequent years [2]. However, due to the long preclinical phase of dementia, it is difficult to

Keenan Sterling and Mengen Xing have contributed equally to this work.

Weihong Song

weihong@wmu.edu.cn

1 Townsend Family Laboratories, Department of Psychiatry, The University of British Columbia, Vancouver, BC V6T 1Z3, Canada

2 Institute of Aging, Key Laboratory of Alzheimer's Disease of Zhejiang Province, School of Mental Health and Kangning Hospital, The Second Affiliated Hospital and Yuying Children's Hospital, Wenzhou Medical University, Wenzhou 325035, China

3 Oujiang Laboratory (Zhejiang Lab for Regenerative Medicine, Vision and Brain Health), Wenzhou 325000, China determine if these associations reflect a causal role for infections in the etiology of dementia or are a consequence of the pathological changes that arise prior to cognitive decline. To address this, Sipilä et al. assessed whether a history of hospital-treated infection is linked to long-term diagnoses of incident dementia in a primary cohort consisting of 260,490 Finnish adults and a replication cohort of 485,708 participants obtained from the UK Biobank with median follow-up periods of 15.4 and 7.7 years, respectively [1]. Hospitalization records for $>900$ different types of infection were obtained and associated with the risk of later developing dementia or specific types of dementia (Alzheimer's, vascular, frontotemporal, and Parkinson's). The results suggest that being hospitalized for any type of infection is associated with an increased risk of developing dementia, with the strongest risk found for vascular dementia. Notably, these findings were then confirmed in follow-up analyses that excluded incident dementia cases occurring within 10 years of the hospitaltreated infection. This method minimized the interference of reverse causation on the effect size (i.e., the effect that preclinical dementia has on disease susceptibility) and strongly supported the notion that infection might play a role in the pathogenesis of dementia.

It has been suggested that the association between infectious diseases and dementia may be specific to certain pathogens or types of dementia. For example, experimental studies have linked infection with the herpes virus to Alzheimer's disease (AD) by showing that $\mathrm{A} \beta$ fibrillization can entrap the viruses, leading to greater $A \beta$ deposition in the brain of an AD transgenic mouse model [3]. However, Sipilä and colleagues found that the risk of developing dementia does not vary substantially with the type of infectious disease (bacterial, viral, parasitic, or fungal) or the capacity of the disease to enter the central nervous 
system (CNS) [1]. On the other hand, a clear dose-response relationship was found between the burden of infection (i.e., the number of infectious episodes) and dementia. Together, the absence of disease specificity and the doseresponse relationship between infectious burden and the incidence of dementia implies that the increased dementia risk may be driven by the inflammatory challenge itself rather than the specific pathogen.

To determine if the involvement of the CNS is necessary for a pathogen to affect the brain, the authors also investigated the risk of developing dementia when analyses were restricted to infections localized outside the CNS (i.e., pneumonia and appendicitis). Although a greater risk was found for CNS infections, peripheral infections were also linked to an increased risk of dementia, including a 1.8-fold greater risk of $\mathrm{AD}$ and a 3.3-fold greater risk of vascular dementia. These findings suggest that systemic infections can invoke an inflammatory response that sufficiently enables peripheral pathogens to contribute to the development of dementia without entering the brain. A potential mechanism behind this could be that peripheral infections trigger an increased production of pro-inflammatory molecules leading to the activation and infiltration of peripheral monocytes into the brain (Fig. 1) [4]. In a mouse model of vascular cognitive impairment, elevated neuroinflammatory signaling in the brain can cause microglial activation and chronic reactive gliosis that contributes to cognitive decline in an astrocyte-dependent manner [5].
Collectively, the results of these studies also indicate that inflammatory mediators of vascular dementia might serve as a common pathological mechanism that underlies the association between infectious diseases and dementia. These findings are particularly relevant given the potential implications for the COVID-19 pandemic. With $>200$ million confirmed cases, including $>4.3$ million deaths already reported by the World Health Organization (https:// covid19.who.int/info), the results of Sipilä et al. highlight the need to explore whether the inflammatory reaction contributes to the development of cognitive dysfunction in COVID-19 patients.

Over the past year, evidence from observational studies has already hinted at a potential link between COVID-19 and dementia. For instance, COVID-19 infections have been found to adversely impact the immune and nervous systems even among patients without neurological symptoms [6], suggesting the potential for long-term cognitive effects. Furthermore, a recent article published in Molecular Neurodegeneration by Dr. Yan-Jiang Wang's research group has provided early insights into the potential impact of COVID-19 on cognitive decline [7]. Focusing their study on the first batch of patients to be discharged from three designated COVID-19 hospitals in Wuhan, China, the authors investigated the cognitive effects of mild and severe COVID-19 infections on cognition in elderly patients after six months of recovery. They recruited 1539 COVID-19 patients, including 238 severe and 1301
Fig. 1 How systemic infections could contribute to dementia. After severe infection, chronically elevated levels of inflammatory mediators could promote monocyte infiltration into the brain. Invading monocytes then release high levels of inflammatory cytokines (1), leading to microglial activation (2) and inducing neuroinflammatory reactive astrocytes. In turn, activated microglia further produce inflammatory cytokines and reduce the clearance of toxic products (3). Reactive astrocytes then cause vasoconstriction and chronic hypoperfusion across the blood-brain barrier (BBB; 4), ultimately leading to neurodegeneration and cognitive dysfunction. Image created with BioRender.com.

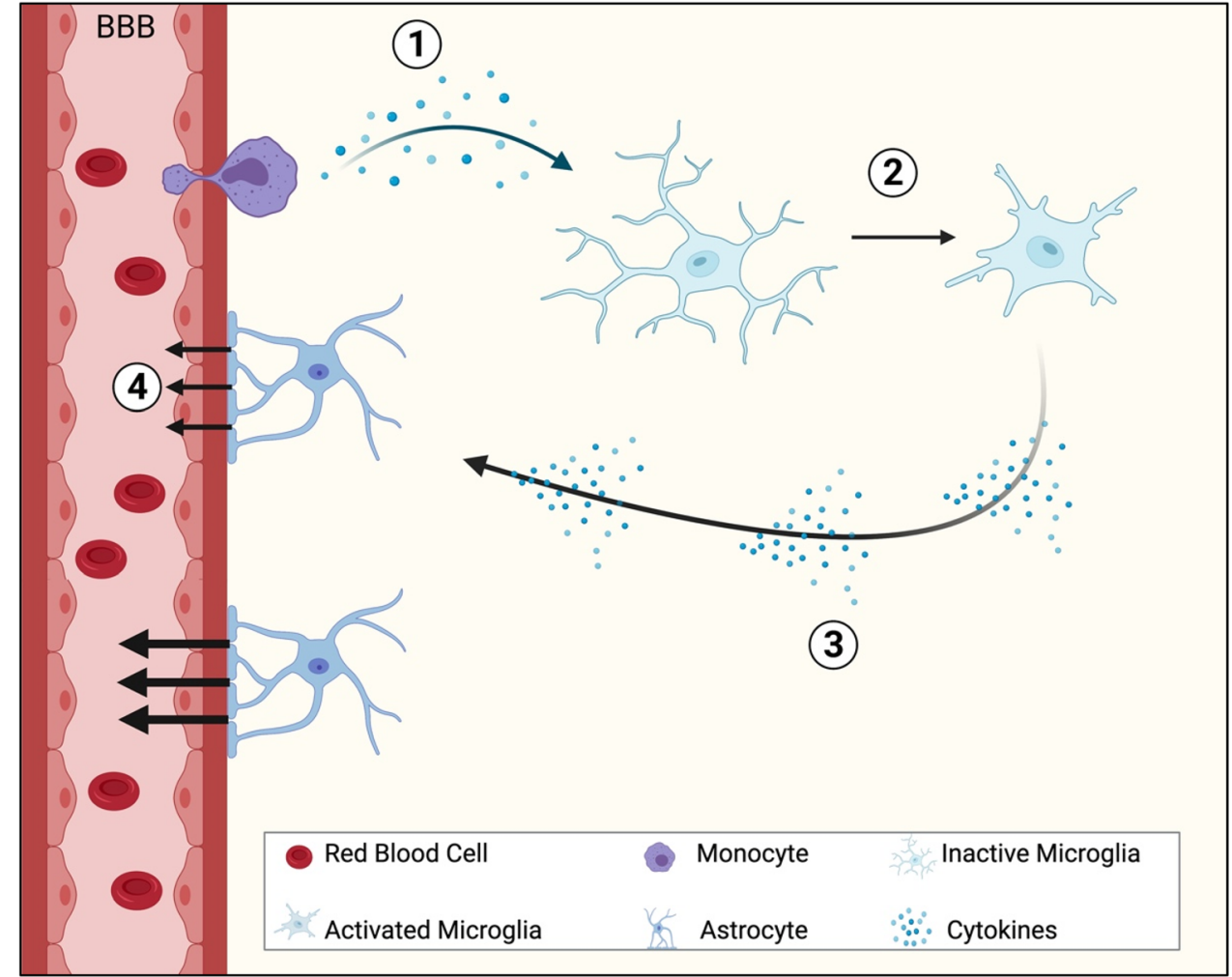


non-severe cases, along with 466 uninfected spouses who were selected as controls. Disease severity was associated with the cognitive status of patients (mild cognitive impairment and dementia) and their longitudinal cognitive decline during the recovery period, and assessments were made using standardized telephone surveys. The results of this study indicated that severe COVID-19 patients were more likely to exhibit cognitive deficits six months following infection when compared to mild patients and controls, with $35.7 \%$ of patients meeting the criteria for cognitive impairment. However, no such differences were found between mild COVID-19 patients and controls, suggesting that experiencing a severe reaction is critical in predisposing patients to cognitive impairment. On the other hand, both severe and mild COVID-19 patients were reported by relatives as exhibiting longitudinal cognitive decline over the six-month recovery period. As such, these findings suggest that elderly COVID-19 patients experienced a rapid decline in cognitive capabilities during postinfection recovery, with stronger effects found after a severe infection.

New research presented at the Alzheimer's Association International Conference (AAIC) 2021 further highlighted other studies currently underway to determine whether COVID-19-related cognitive deficits persist in the long term and if they are associated with biomarkers of particular types of dementia. For example, preliminary findings on a cohort of 300 COVID-19 patients from Argentina suggested an increased risk of cognitive deficits among patients assessed between three and six months after infection. Importantly, participants will continue to be tracked for three years to monitor long-term changes. Another study currently underway at New York University-Langone Health is investigating the blood of $>300$ COVID-19 patients for biomarkers of AD (Neergaard L. Research looks for possible COVID tie to later Alzheimer's. https://medicalxpress.com/news/2021-07-covid-alz heimer.html. 2021). Patients with incident neurological symptoms were found to exhibit elevated levels of certain biomarkers linked to neuroinflammation and dementia, including C-reactive peptide, glial fibrillary acid protein, total tau, and phosphorylated tau (pTau-18). These markers suggest that COVID-19 may induce pathological changes associated with dementia, such as inflammation-related neuronal injury and glial activation.

Overall, a growing body of research seems to support the notion that systemic infections contribute to the etiology of dementia. The report by Sipilä and colleagues was the first large-scale multicohort study to incorporate a wide range of infectious diseases and specifically examine the long-term effects of infection on dementia risk. Their findings provide strong support for the link between infection and dementia and suggest that inflammatory mediators of vascular dementia might serve as a common pathological mechanism underlying this association [1]. Notably, the generalizability of the infection-dementia association found in this study indicates that a similar pathology may extend to patients with COVID-19. In agreement, the report by Dr. Wang and colleagues found that elderly COVID-19 patients are more likely to exhibit cognitive deficits as early as 6 months following a severe infection [7]. While more longitudinal studies are needed to assess the long-term consequences of COVID-19 on cognitive function, the preliminary results recently reported at the AAIC also indicate that COVID-19 patients may be at a higher risk for dementia (Neergaard L. Research looks for possible COVID tie to later Alzheimer's. https://medicalxpress.com/news/2021-07-covid-alzheimer. html. 2021). These findings are particularly concerning for the long-term health of the elderly and people with $\mathrm{AD}$, the most vulnerable population during the COVID-19 pandemic [8]. Future studies are needed to investigate the causal mechanism whereby an infection may trigger dementia and identify the time window when patients are more at risk as this might help provide specific targets for therapeutic intervention.

Conflict of interest The authors declare that they have no conflict of interest.

\section{References}

1. Sipilä PN, Heikkilä N, Lindbohm JV, Hakulinen C, Vahtera J, Elovainio M. Hospital-treated infectious diseases and the risk of dementia: A large, multicohort, observational study with a replication cohort. Lancet Infect Dis 2021, 21: 1557-1567.

2. Tate JA, Snitz BE, Alvarez KA, Nahin RL, Weissfeld LA, Lopez $\mathrm{O}$, et al. Infection hospitalization increases risk of dementia in the elderly. Crit Care Med 2014, 42: 1037-1046.

3. Eimer WA, Vijaya Kumar DK, Navalpur Shanmugam NK, Rodriguez AS, Mitchell T, Washicosky KJ, et al. Alzheimer's disease-associated $\beta$-amyloid is rapidly seeded by Herpesviridae to protect against brain infection. Neuron 2018, 100: 1527-1532.

4. D'Mello C, Le T, Swain MG. Cerebral microglia recruit monocytes into the brain in response to tumor necrosis factoralpha signaling during peripheral organ inflammation. J Neurosci 2009, 29: 2089-2102.

5. Saggu R, Schumacher T, Gerich F, Rakers C, Tai K, Delekate A, et al. Astroglial NF-kB contributes to white matter damage and cognitive impairment in a mouse model of vascular dementia. Acta Neuropathol Commun 2016, 4: 76.

6. Qin YY, Wu JF, Chen T, Li J, Zhang GL, Wu D, et al. Long-term microstructure and cerebral blood flow changes in patients recovered from COVID-19 without neurological manifestations. J Clin Invest 2021, 131: 147329.

7. Liu YH, Wang YR, Wang QH, Chen Y, Chen X, Li Y, et al. Postinfection cognitive impairments in a cohort of elderly patients with COVID-19. Mol Neurodegener 2021, 16: 48.

8. Zhang Q, Song W. The challenges of the COVID-19 pandemic: Approaches for the elderly and those with Alzheimer's disease. MedComm (Beijing) 2020, https://doi.org/10.1002/mco2.4. 